\title{
Bound versus Free Water in Dormant Apple Buds-A Theory for Endodormancy
}

Miklos Faust and Dehua Liu

Fruit Laboratory, Beltsville Agricultural Research Center Agricultural Research Service, Beltsville, MD 20705

Merle M. Millard

Environmental Chemistry Laboratory, Beltsville Agricultural Research

Center, Agricultural Research Service, Beltsville, MD 20705

G.W. Stutte

Department of Horticulture, University of Maryland, College Park, MD 20742

Additional index words. Malus domestica, chilling, magnetic resonance imaging, thidiazuron

Abstract. Intact apple (Malus domestica Borkh.) buds were examined by magnetic resonance imaging (MRI). MRI did not excite water in unchilled apple buds and could not image it. When chilling was satisfied, images were produced. We interpret this difference to mean that water is in bound and/or structured form in dormant apple leaf buds before the chilling requirement is satisfied. Conversion of bound to free water occurred equally in the low-chilling-requirement cultivar Anna and the high-chillingrequirement cultivar Northern Spy only after 600 and 4000 hours of chilling, respectively. It appears that processes involved in satisfying chilling requirement are also converting water in buds from bound to free form. Absence of free water in dormant buds during the winter signifies endodormancy, whereas when the water is in free form, buds are ecodormant. Thidiazuron, a dormancy-breaking agent, applied to partially chilled buds is instrumental in converting water to the free form within 24 hours. Summer-dormant buds contain free water, and they could be classified only as paradormant. Based on proton profiles, ecodormant and paradormant buds cannot be distinguished but endodormant buds can be readily identified.

Nondestructive imaging of plant tissues by magnetic resonance imaging (MRI) is a useful method to assess the water status of certain plant organs. Soft-tissue-contrast in MRI imaging is primarily due to differences in proton relaxation times. Relaxation characteristics are related to molecular motion. Theories on this subject were recently reviewed (Wehrli, 1988). The two relaxation processes can be described by the longitudinal ( $\mathrm{Tl}$ ) and transverse (T2) relaxation times and have been used to study water in plant tissues (Chen et al,. 1978; Stout et al., 1978).

The average rate at which molecules reorient in a magnetic field is related to molecular size. Small molecules, such as water, reorient more rapidly and relax more slowly than larger molecules, which reorient slowly and relax much faster. Free water relaxes slowly (long T1 and T2 times) and gives a sharply contrasting MR image, but, water bound to or perturbed by macromolecules (called structured water) relaxes considerably faster (short $\mathrm{T} 1$ and $\mathrm{T} 2$ times) and does not show on the MR image (Fullerton, 1988).

Received for publication 17 Oct. 1990. The cost of publishing this paper was defrayed in part by the payment of page charges. Under postal regulations, this paper therefore must be hereby marked advertisement solely to indicate this fact.
Water fractions may change in tissues as the tissue function changes. Thus, images obtainable from the tissues may also change accordingly.

Temperate-zone fruit trees, including apple, require certain amounts of chilling before they can resume growth. There are models that predict chilling requirement. However, little is known about the physiological and biochemical events that mark the

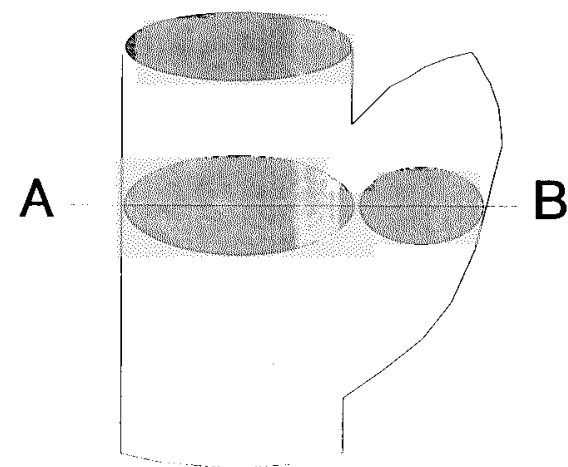

Fig. 1. Schematic illustration of imaging plane of apple buds. The two circles, transected by the line A-B, correspond to the images of MRI. Occasionally, a graph is superimposed on the image between A and B representing the relative MR signal intensity for each pixel. 


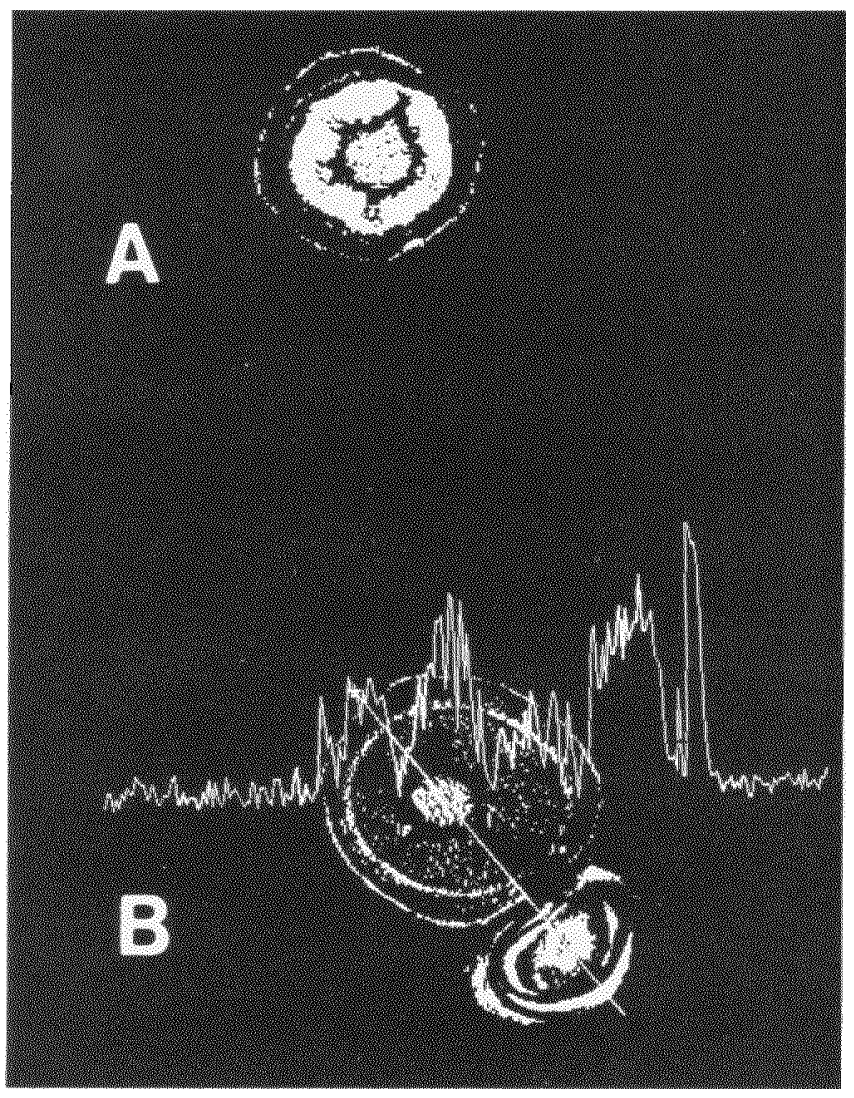

Fig. 2. Magnetic resonance images of leaf buds of 'Anna' apple (A) after $200 \mathrm{~h}$ and (B) after $600 \mathrm{~h}$ of chilling. Note that (A) has only the stem section image and (B) shows both the the stem andbud section images.

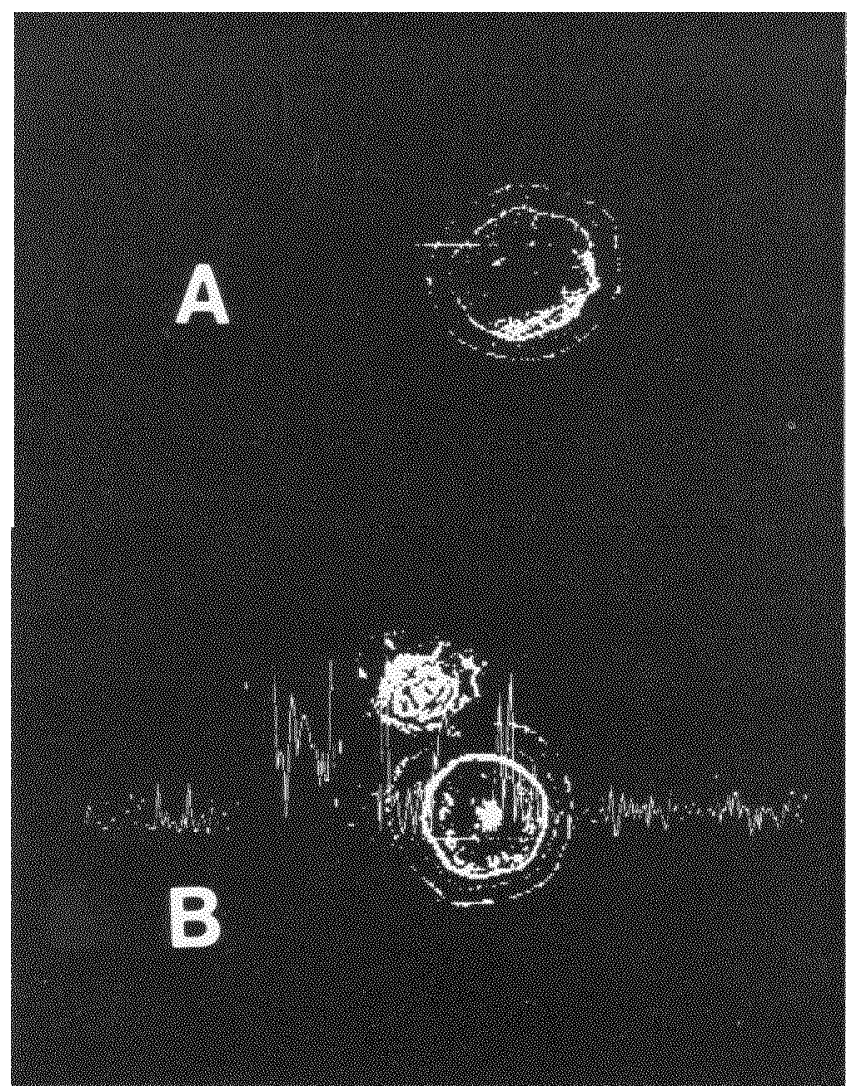

Fig. 3. Magnetic resonance images of leaf buds of 'Northern Spy' apple (A) after $1000 \mathrm{~h}$ and (B) after $4000 \mathrm{~h}$ of chilling. Similarly to 'Anna', (A) shows only the stem section image, whereas (B) has both stem and bud images. end of the chilling period (Faust, 1989). Lang et al. (1985) proposed a unified terminology for dormancy. They distinguished between para-, endo-, and ecodormancy depending on factors regulating dormancy by physiological factors originating outside or inside of the affected structure, or by the environment, respectively. Endodormancy in fruit tree buds is thought to be released by chilling; thereafter, the buds are in ecodormancy until growth resumes (Faust, 1989). Paradormant buds during summer dormancy can be forced to grow by removing the terminal bud of the shoot. Such operation does not force resumption of growth in buds that are endodormant. Resumption of growth also can be forced by cytokinin-type compounds such as benzyladenine in summer-dormant buds (Broom and Zimmerman, 1976) and by thidiazuron (TDZ) in buds that may be characterized as paradormant (e.g. before chilling is initiated or during summer dormancy) (Steffens and Stutte, 1989, Wang et al., 1988).

Here, we report the water status of apple buds, determined by MRI, during chilling, summer dormancy and forcing by TDZ.

'Anna', a low-chilling-requiring cultivar, and 'Northern Spy', a high-chilling-requirement cultivar, were used for the studies. Shoots, grown during the summer, were cut late in October and stored at $4 \mathrm{C}$ in a cold room. Samples were removed after specified chilling hours had been imposed. Each shoot, after removal from the cold, was placed in distilled water for 20 to $24 \mathrm{~h}$, at room temperature, before MRI analysis. Buds of 'Anna' were treated with TDZ by painting a 100ppm solution on the buds after removal from storage; these buds were kept at room temperature for $24 \mathrm{~h}$, similar to untreated buds as described.

Summer-dormant buds to be imaged were prepared on 25 July 1990, by removing the terminal buds in the orchard from some shoots and leaving others intact. Sample shoots of both types, without terminals and with set terminal buds, were collected 1 Aug. 1990. By sample collecting time, on shoots where the terminal bud had been removed, the top two lateral buds started to break. MRI was performed on buds below those breaking and buds at comparable locations on shoots with terminal buds intact. MR images of replicate samples were remarkably similar. Leaf buds were used for all imaging.

Shoots were placed in 10-mm MRI tubes for imaging with a Bruker MSL, $400 \mathrm{MHz}$ (9.4T) microimaging instrument (Broker Instr. Co., Billerica, Mass.). Conventional spin echo 2DFT images of matrix size $256 \times 256$ were obtained with spatial resolution of 58.5 $\times 58.5 \times 720 \mu \mathrm{m}$. Method was similar to that described by Kuhn (1990). In all images, the TE was $14 \mathrm{~ms}$ and the TR was 514 ms. First, images were taken with fewer being averaged for rapidity of examination. This procedure resulted in excessive noise in the images, but allowed rapid replication of samples. Then, selected samples were imaged with more being averaged. Due to signal noise considerations, between 100 and 


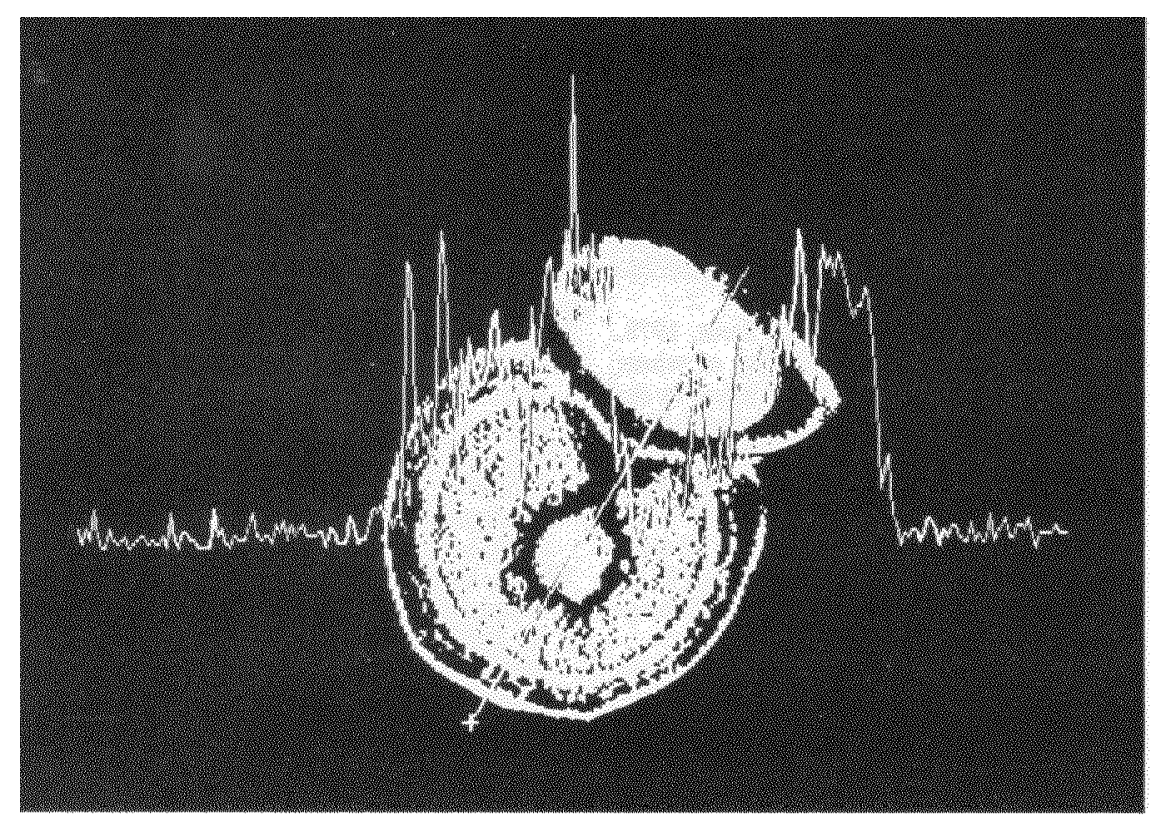

Fig. 4. Magnetic resonance images of leaf buds of 'Anna' apple treated with 100 ppm TDZ after 200 $\mathrm{h}$ of chilling. The image should be compared with Fig. 2A (untreated at the same chilling level) and Fig. 2B (a fully chilled bud).

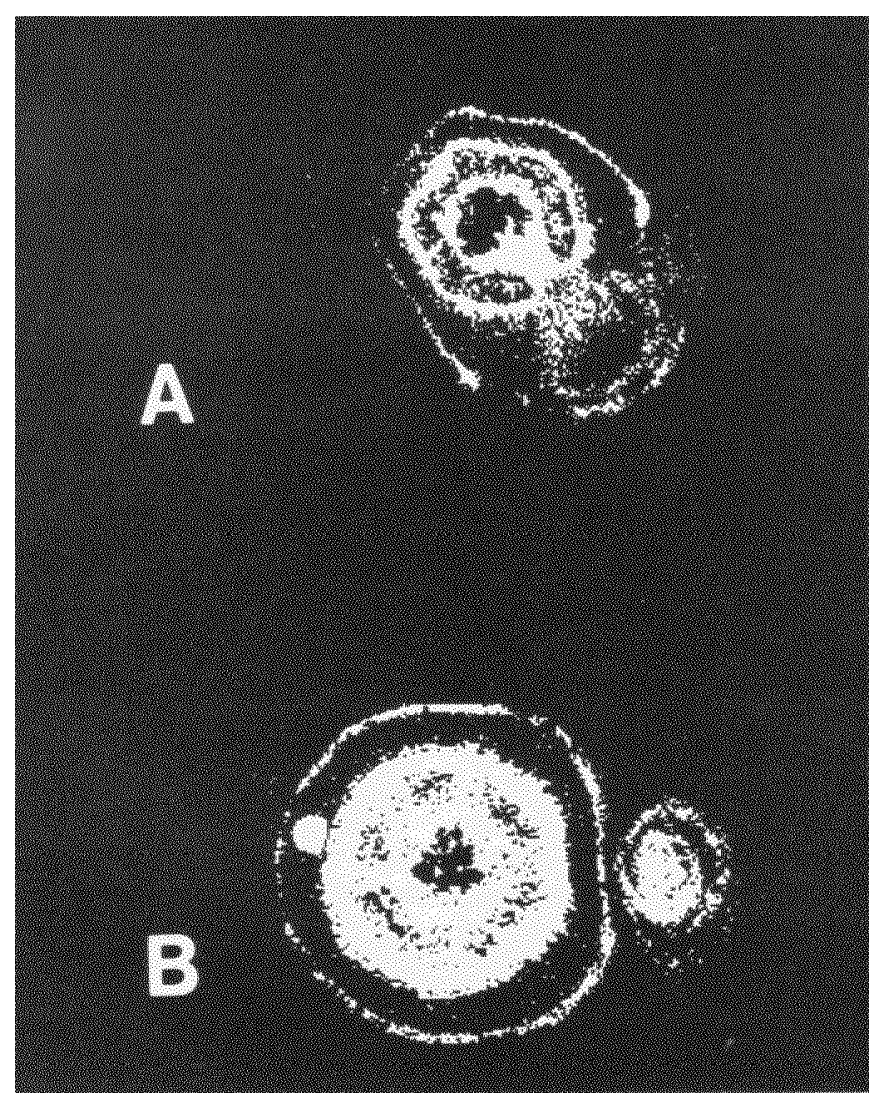

Fig. 5. Magnetic resonance image of summer-dormant buds of 'Anna' apple. (A) Image of lateral bud with intact terminal bud. (B) Image of lateral bud on a shoot where terminal bud was removed 1 week before imaging.

512 averages were taken, resulting in data acquisition times of up to $24 \mathrm{~h}$. Image slices were taken perpendicular to the axis of the shoot about at the midpoint of the bud (Fig. 1). Taking the image slice higher or lower
According to the proton profiles for both cultivars, sufficient free water to produce an image was present only in the stem before the chilling requirement was satisfied (Figs. $2 \mathrm{~A}$ and $3 \mathrm{~A}$ ). Buds produced no image at this time. Free water in the bud was readily observable and created images in 'Anna' after $600 \mathrm{~h}$ of chilling (Fig. 2B) and in "Northern Spy' after $4000 \mathrm{~h}$ of chilling (Fig. 3B). Intermediate stages before the chilling requirement was satisfied showed only traces of free water (images not presented). The moisture content of buds, determined by dry weight, was $49 \%$ before and $59 \%$ after chilling was satisfied and the difference was statistically not significant. This result indicates that, in the unchilled, endodormant buds, the water is in a form that creates $\mathrm{T} 1$ or $\mathrm{T} 2$ times that are too short to form an image. We interpreted this result to mean that in endodormant buds, water is in bound or structured form. This interpretation further implies that endodormancy is a stage of bud development when water is in a bound or structured form. This situation contrasts with that of chilled buds, in which sufficient quantity of water is in free form to create images. So far, we do not know, but do suspect from application of TDZ, that a substantial part, but not all, of the water is in a free form. Regardless of the portion of water that is in free form, chilled buds differs greatly from endodormant buds as far as the form of water is concerned.

Chen et al. (1978), in winter wheat crowns, observed a significant decrease in relaxation times of T1 and T2 with nuclear magnetic resonance (NMR) after 7 weeks of acclimation. T2 time for tissue culture aggregates of wheat was much longer than that in fully hardy crowns (Stout et al. 1978). Stout et al. (1978) observed two populations of water in ivy bark with NMR, one with short T1 and $\mathrm{T} 2$ times ( $\approx 32 \%$ to $40 \%$ of the water) and another with long T1 and T2 times $(\approx 60 \%$ to $68 \%$ of the water). They believed that the larger quantity of water, which had the longer T2 time, was intracellular water, and the water that had short T2 time was associated with cell walls. We do not know the degree of dormancy in wheat crowns or in ivy bark, but we believe that these studies indicate that at least part of the water can be in bound/ structured form in hardened and partially dormant plant tissues-in contrast to the presence of free water (long T2 time) in tissue culture aggregates, which represent actively growing tissue.

The outside layer of bark, and an inner ring, where the cambial layer and/or the younger areas of xylem are, contain considerable free water even before chilling requirement of buds is satisfied. If the absence of free water is considered as a criterion for endodormancy, as we advocate, tissues of the stem are not endodormant.

TDZ treatment changed the bound/structured water-free water relationship within 24 $\mathrm{h}$ in 'Anna', and the buds became visible on MRI images with a higher-intensity proton signal than satisfying the chilling requirement can produce (Fig. 4). Images of buds without TDZ were the same as shown in Fig. 
2A. TDZ is not effective during endodormancy of other cultivars to induce growth (Steffens and Stutte, 1989) and needs to be applied in late fall, before chilling is initiated, or to partially chilled buds when a substantial part of the chilling requirement has been satisfied. TDZ is also effective in breaking summer dormancy of apples (Wang et al., 1986). From these limited experiences, it appears that TDZ is effective in paradormant buds to force growth or in buds that are near to the end of their endodormancy. Because TDZ is effective after 200 $h$ of chilling (less than half the normal requirement) in 'Anna' buds in converting water to free form, which is required for resumption of growth, it appears that 'Anna' buds are not in as deep dormancy as those of 'Northern Spy', which require $80 \%$ or more of their chilling requirement to be satisfied before TDZ is able to break dormancy (Steffens and Stutte, 1989). Thus, we may be looking not only at a difference in length in endodormancy, but also a difference in the depth of endodormancy. TDZ appears to be a useful tool to evaluate this aspect.

Summer dormant buds contain free water (Fig. $5 \mathrm{~A}$ and B). When the terminal bud is removed and the top buds of the shoots are activated, the proton profile of the still-dormant lateral bud (Fig. 5B) is somewhat more intense than that of the lateral bud associated with an intact terminal bud of the shoot (Fig. 5A). Nevertheless, based on proton profile, the dormant buds collected during the summer contained free water and their proton profile was definitely different from the buds that were endodormant.

The xylem proton profile of shoots is much less clear than similar profiles in endodormant or paradormant buds. Intensity of free water of xylem of the the shoot with the terminal bud removed and the top two or three buds activated (Fig. 5B) shows much higher free water concentration than the xylem of the shoot with the terminal bud and with all the buds dormant (Fig. 5A). In fact, the xylem proton profile of shoots activated to grow by removing the terminal bud (Fig. $5 \mathrm{~B}$ ) is as strong as the xylem proton profile of stems on which buds were activated by TDZ (Fig. 4). This pattern contrasts with the relatively weak proton profile of the xylem of the chilled or unchilled dormant buds (Figs. $2 \mathrm{~B}$ and $3 \mathrm{~A}$ and $\mathrm{B})$. However, occasionally we had higher proton profile in the xylem even in shoots with endodormant buds (Fig. 2A). Although activation of buds either by TDZ or by removing terminals over paradormant buds may increase free water in the xylem, we do not know the importance of free water in the xylem of endodormant or paradormant shoots without further research.

In considering winter buds, bound water signifies endodormancy. As chilling is completed, the buds have free water. They are ready to grow, but they are still dormant because the environment is not conducive to growth. Such buds are classified ecodormant. Based on proton profile, summer-dormant (paradormant) buds are not different from those in ecodormancy. Thus, based on proton profiles, we cannot distinguish between ecodormant and paradormant buds, but we can definitely separate endodormant buds from the others.

\section{Literature Cited}

Broom, O.C. and R.H. Zimmerman. 1976 Breaking bud dormancy in tea crabapple with cytokinins. J. Amer. Soc. Hort. Sci. 101:2830.

Chen, P.M., L.V. Gusta, and D.G. Stout. 1978. Changes in membrane permeability of winter wheat cells following freeze-thaw injury as determined by nuclear magnetic resonance. Plant Physiol. 61:878-882.

Faust, M. 1989. Physiology of temperate zone fruit trees. Wiley, New York.

Fullerton, G.D. 1988. Physiologic basis of magnetic relaxation, p. 36-55. In: D. Stark and W.G. Bradley, Jr. (eds.). Magnetic resonance imaging. C.V. Mosby, St. Louis.

Kuhn, W. 1990. Microscopy fundamentals, limits and possible applications. Angewandte Chemie Intl. 29:1-112.

Lang, G.A., J.D. Early, N.J. Arroyave, R.L. Darnell, G.C. Martin, and G.W. Stutte. 1985. Toward a reduced universal terminology. HortScience 20:809-811.

Steffens, G.L. and G.W. Stutte. 1989. Thidiazuron substitution for chilling requirement in three apple cultivars. J. Plant Growth Regulat. 8:301308.

Stout, D.G., P.L. Steponkus, and R.M. Cotts. 1978. Nuclear magnetic relaxation times and plasmolemma water exchange in ivy bark. Plant Physiol. 62:636-641.

Wang, S.Y., G.L. Steffens, and M. Faust. 1986. Breaking bud dormancy in apple with plant bioregulator, thidiazuron. Phytochemistry 25:311-317.

Wehrli, F.W. 1988. Principles of magnetic resonance, p. 3-23. In: D. Stark and W.G. Bradley, Jr. (eds.). Magnetic resonance imaging. C.V. Mosby, St. Louis. 